\title{
The ARISE Reconfigurable Instruction Set Extensions Framework
}

\author{
Nikolaos Vassiliadis, George Theodoridis, and Spiridon Nikolaidis \\ Sect. of Electronics \& Computers, Depart. of Physics \\ Aristotle University of Thessaloniki \\ Thessaloniki, Greece \\ e-mail: nivas@physics.auth.gr
}

\begin{abstract}
In this paper, we introduce the ARISE framework for the systematic extension of typical processors with the necessary infrastructure to support arbitrary number and type of reconfigurable hardware units. ARISE extends the microarchitecture of the processor with an interface to allow the coupling of the hardware units. Furthermore, the instruction set of the processor is extended with instructions which expose to the programmer/compiler the full control of the interface. This control includes the configuration of operations on the hardware units, execution of these operations, and communication of data between the processor and the units. The new instructions are incorporated without the need to redesign the processor instruction set architecture. To evaluate our proposal a model of an ARISE extended MIPS processor has been designed. Using a turbodecoder algorithm as benchmarking application a simulation of the ARISE model has been performed. Performance results show impressive application speedups up to $\mathbf{x} 7.5$.
\end{abstract}

\section{INTRODUCTION}

Instruction set customization is an effective way to improve performance over a certain set of applications (application domain). Critical portions of the application can be more efficiently executed on Custom Computing Units (CCUs). While the base instruction set serves as the bulk of the flexibility used to implement any application, the customized instructions utilize the CCUs to enhance performance over the target application domain. Furthermore, providing to the CCUs the capability to reconfigure their functionality, high degrees of flexibility are gained. Reconfigurable units provide dynamic instruction set extensions offering the adaptation of the system to the targeted application.

The process of designing such a hybrid system, except from designing the CCUs itself, is divided in two steps: first provide an interface between the processor and the CCUs and second expose to the architecture the control of the new system. Although a number of such systems have been presented in the literature most of them use an ad-hoc design approach to couple the CCUs to the processor. In addition, most systems are designed based on the needs of the attached CCUs excluding this way modularity and making difficult the extension of the system with new types of CCUs. Finally, in most cases the approaches presented in the literature are suffering from limitations which could reduce performance. Such limitations are the number of parameters which the CCU can access (parameters limitation), the opcode space available to encode operations performed in the CCUs (opcode space explosion) etc.

In this paper we present the AUTH Reconfigurable Instruction Set Extension (ARISE) framework. The framework extends a typical processor with: 1) a micro-architectural interface which is used to couple CCUs to the processor and 2) a set of instruction set extensions to control the interface. These extensions are one-time performed to a processor to create an ARISE machine. After that, an arbitrary number of CCUs can be attached to the machine. Moreover, the CCUs complexity can vary from hardwired to reconfigurable units with multiple contexts of configurations. The instruction set extensions exposes to the programmer/compiler all necessary instructions required to control the configuration of operations in the CCUs, their execution, and the communication of arguments between CCUs and the processor. Furthermore, using a buffering technique ARISE overcomes the parameters limitation while exploiting at the same time the complete register file bandwidth. ARISE also deals with the opcode space explosion by dynamically assigning opcodes to operations.

To evaluate the proposal, a model of an ARISE extended MIPS processor was designed. A turbodecoder application was implemented in the ARISE machine and simulations was performed to estimate the performance of the machine and identify any possible bottlenecks. Results indicate that the ARISE machine is able to speedup the execution of the application by a factor of 7.5.

The rest of the paper is organized as follows. Section II gives a general description of the ARISE framework and discusses in detail the general organization of an ARISE machine, the ARISE micro-architectural interface and the ARISE instruction set extensions. Furthermore, the procedure which must be followed to program an ARISE machine is also described. Section III presents the design of an ARISE evaluation machine based on a MIPS core processor. Experimental results are presented in section IV. Section V discusses some related work while we conclude in Section VI. 


\section{GENERAL DESCRIPTION}

\section{A. Organization}

The main components in an ARISE machine (depicted in Figure 1) are the Core Processor and a one-time performed micro-architectural extension, entitled ARISE Interface (AIF). After this extension has been performed, an arbitrary number of CCUs can be attached to the machine utilizing the ARISECCU Wrappers (ACW). The complexity of the CCUs can vary from simple hardwired to complex partially reconfigurable units with multiple contexts of configurations. In the default deployment of an ARISE machine the AIF accesses the Memory Hierarchy to provide data and configuration bits to the CCUs through the standard memory interface of the processor. Alternatively, dedicated configuration memory and/or dedicated memory ports can be used to allow ARISE configuration and/or execution concurrently with processor operation.

Instructions are fetched from the instruction cache and an initial decoding is performed by the ARISE Instruction Decoder (AID) to determine if they are regulars or ARISE instructions. Instructions which are part of the standard processor ISA are issued without modification to the processor. In the case of an ARISE instruction the AID role is twofold. Firstly it forwards specific fields of the instruction word to the AIF. These fields are encoding a second level of ISA, the ARISE ISA (discussed in the following), which is used to control the configuration and execution of ARISE operations in the CCUs. Secondly, the AID generates an appropriate emulation instruction which is issued to the processor. The scope of this instruction is to force the processor to operate in a mode which satisfy the AIF requirements (read/write register file, resolve data hazards etc.). In this way, a processor can be extended by the ARISE without modification and redesign of the processor ISA.

The AIF is further subdivided into the ARISE Control Unit (ACU), the Opcode-to-Address Table, and the Input/Output Buffer. Pieces of the initial application code can be accelerated by executing them in the CCUs as ARISE operations (ARISE operations can be as complex as complete kernels or as simple as a data-flow with a few nodes collapsed in a single operation). The ARISE ISA exposes to the programmer/compiler the required infrastructure to configure the CCUs and execute ARISE operation on them. The ACU decodes the ARISE ISA and appropriately drives the other ARISE components. In addition, it has access to the processor $\mathrm{CU}$ to return status signals and stall the pipeline to support multi-cycle ARISE instructions. The other ARISE components are presented in more detail in the following.

\section{B. The Opcode-to-Address Table}

In an ARISE machine CCUs are identified by an address. This address is configured in the ACW during $\mathrm{CCU}$ deployment and can point to a simple hardwired or to a reconfigurable CCU. Moreover, a range of addresses can be assigned in a CCU its one pointing to a specific configuration context or even to one of the different operations supported by the CCU. This address can be of any size allowing the support of arbitrary ARISE operations. However, the ARISE follows the tightly-coupling approach to reduce the communication overhead between the processor and the CCUs. To be able to call ARISE operations with no overhead they are encoded to an opcode in the instruction word of the processor. However, this approach raises an opcode space explosion problem that limits the number of supported operations.

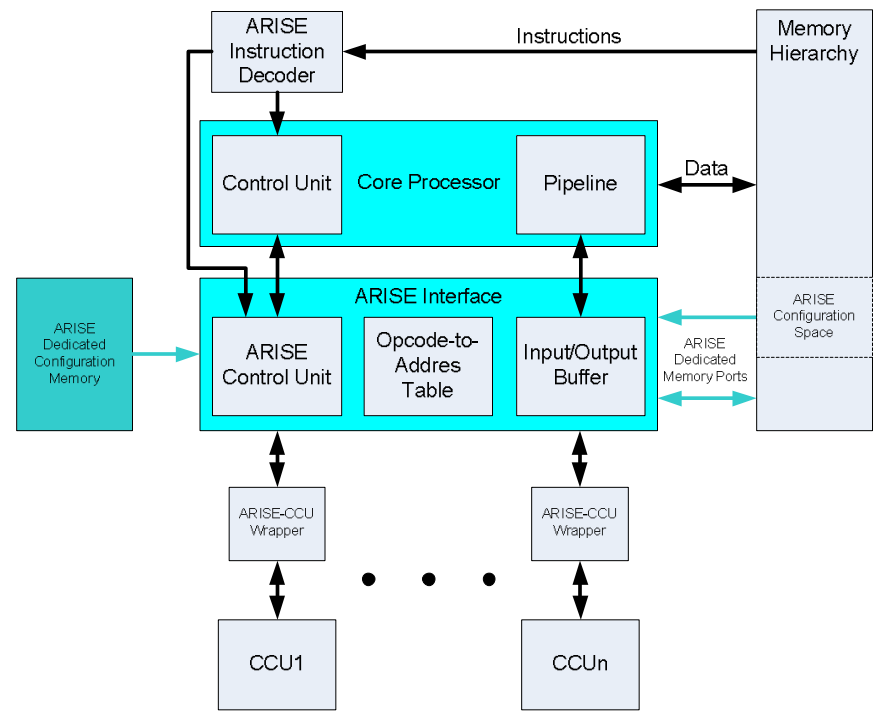

Figure 1. General ARISE machine organization.

To overcome this problem ARISE uses the Opcode-toAddress Table. This table stores the mapping between the opcode of an ARISE operation and the address of the CCU in which the operation has been assigned. The ARISE ISA provides instructions to dynamically remap opcodes to addresses. After such a mapping has been established, an operation can be implicitly called through its assigned opcode. This way the same opcode can be assigned to different operations across the application space. Thus, an almost arbitrary opcode space is provided, eliminating the opcode explosion problem. In addition, since opcodes are not univocally assigned to operations, these operations can be implemented independently in the CCUs and ported later to the ARISE machine preserving modularity and compatibility with other ARISE machines.

\section{The Input/Output Buffer}

Communication of data between the core processor and the AIF is performed through the Input/Output Buffer (IOB). Exploiting the ARISE ISA any number of data (limited only by the size of the IOB) required for an ARISE operation or produced by such an operation can be passed from/to the register file to/from the AIF. For this reason the IOB is used as a temporary storage space. The IOB is configured to utilize the complete register file bandwidth (this is all the read/write ports) reducing this way the communication overhead to the minimum possible (Not considering the case to increase the number of input/output register file ports). To achieve this without modifying the processor ISA the IOB is not addressable outside the ARISE.

Figure 2 presents the IOB organization. The IOB is subdivided in the Input and the Output Buffers which are very 
similar in their organization and their operation. The Input Register Bank stores the input arguments of the operation and provides their values to the CCUs while the Output Register Bank receives results from the CCUs and stores the output arguments of the operation. Since the IOB is not addressable outside the ARISE the Address Generation Units are responsible for the addresses generation, based on the received ARISE instruction, of the banks where the operation arguments will be stored (inputs) or are stored (outputs).

Whenever an ARISE operation requires or provides more arguments than the register file supports a sequence of data moving operations is performed. The arguments are stored in successive places in the banks. In order to generate the address where the next arguments will be stored/are stored the address unit increments the address of the previous argument by an index. For the input bank this index is equal to the number of the register file read ports. Similarly, the index value for the output bank is equal to the number of the register file write ports. To implement an operation in a CCU only the place where the arguments are expected or are provided must be declared in order for the programmer/compiler to generate the appropriate sequence of operations which will move the data. This way, the IOB is exploited in order to overcome the argument limitation problem of operations implemented in the CCUs.

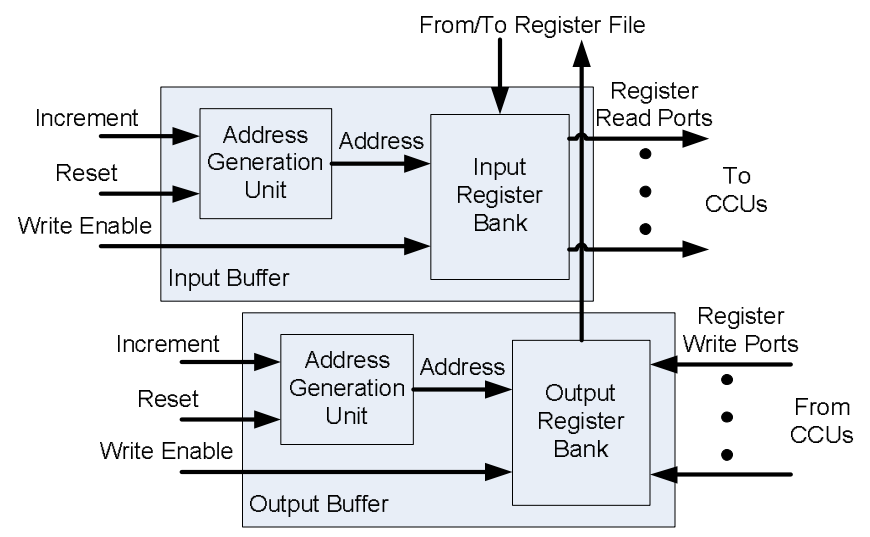

Figure 2. The Input/Output Buffer Organization

\section{The ARISE-CCU Wrappers}

ACWs are extra ARISE components which are not necessarily part of the initial ARISE machine. They are utilized in order to attach CCUs to the AIF and they are configured during deployment to best match the needs of the attached CCU. Their role is twofold. The first is to map a range of addresses to a CCU. As already stated these addresses identify the different operations which can be implemented in the CCU. The second is to provide storage space for the configuration bits which hold ARISE specific information (like cycles required for the completion of an operation, type of the operation etc) for each one of the operations that the $\mathrm{CCU}$ supports.

\section{E. The ARISE ISA}

In order to target the ARISE machine the ARISE ISA exposes to the programmer instructions required to control the configuration of operations in the CCUs, their execution, and the communication of arguments between CCUs and the core processor. The final form of the ARISE ISA can vary both in the type of instructions and the type of instruction parameters, since it can be configured at design time to more accurately match the characteristics of the core processor under extension. The instruction list of the ARRISE ISA (excluding trivial instructions like initialization, no operation etc.), is as follows:

\section{1) Configuration Instructions:}

confopc2addr $<$ opcode $>,<$ ccu_address $>$ : This instruction stores the mapping between the opcode of an ARISE operation and the address of a CCU to the Opcode-to-Address Table. The ACU locks the address of the CCU and waits for configuration bits to be provided. This instruction can be used at the boot-up of the processor in order to map opcodes to CCUs. This way the modularity of the machine is achieved since CCUs can be ported later or even substituted with others just by re-assigning their addresses to a new opcode. In addition, using this instruction dynamically during the execution of an application the same opcode can be re-assigned to a different operation based on the needs of the executed code fragment. So a "virtually" unlimited opcode space is provided.

In the case when two instruction arguments can not be supported by the architecture (because of register file port limitation or because of memory mapped arguments) the following instructions can be used instead:

- $\quad$ confopc $<$ opcode>: The opcode value is stored in the table and the ACU locks the table index expecting the CCU address for this opcode.

- $\quad$ confaddr $<$ ccu_address $>$ : The address value is stored in the table in the place implicitly defined by the ACU based in the previous confopc instruction.

confld <start_address $>$, <end_address $>$ : This instruction initiates the download of configuration bits from the configuration memory to a CCU. The start_address and end_address values provide the addresses in the memory where the configuration bitstream starts and ends respectively. The ARISE then generates the intermediate addresses where the other parts of the bitstream are stored and issues a sequence of memory load operations to download it. Until the downloading process is over the processor is stalled. This way a CCU with any type and size of configuration can be attached at any time to an ARISE machine. Moreover, partially reconfigurable CCUs are also supported. If two instruction arguments can not be supported by the architecture a sequence of instructions: confld_start < address $>$ and confld_end <address $>$ can be used instead. A variation of this instruction to support configuration concurrently with processor operation is also provided and is presented below.

confld_conc <start_address $>$, <end_address $>$ : If a dedicated access to the configuration memory is granted to the ARISE, this instruction is used to initialize the downloading of the configuration bitstream without stalling the processor. Thus, the downloading of the configuration bits for an operation can be scheduled well before the usage of the operation in order to hide the cost. One argument version of 
this instruction (confld_start, confld_end_conc) is also available.

\section{2) Execution Instructions:}

execa $<$ opcode $>,<\operatorname{dest1}>, . .,<\operatorname{destN}>,<$ src1 $>, . .,<$ srcM $>$ : This instruction calls the execution of an ARISE operation (encoded in opcode). The input arguments are moved from the src1...srcM registers of the processor register file to the Input Buffer while output arguments are moved from the Output Buffer to the dest1...destN registers of the processor register file. If the operation is multi-cycle the processor is stalled until its completion. The number of cycles required for the completion is part of the configuration bitstream. The operation of this instruction is identical to typical arithmetic/logic operations found in RISC machines. The numbers $\mathrm{M}$ and $\mathrm{N}$ are configured to be equal to the numbers of read and write ports of the processor register file respectively to utilize all available data bandwidth.

execa_conc $<$ opcode $>, \quad<$ src1 $>, . . . ., \quad<$ srcM $>$ : This instruction is similar to the previous with the difference that allows concurrent operation of the processor and CCUs. However, this mode of operation can cause data inconsistency and a synchronization mechanism is required. The instruction doesn't include any output arguments which can be obtained with the use of a movfa instruction (presented below). This movfa instruction acts as the implicit synchronization instruction. The programmer/compiler requests results of an ARISE operation with a movfa instruction. If the ARISE has not yet completed the operation then it stalls the processor breaking concurrent operation. Scheduling of the movfa instruction close to the code which uses the result of the ARISE operation, can hide cycles required by a multi-cycle ARISE operation by allowing concurrent operation for the maximum possible time.

\section{3) Move Instructions:}

movta <src1>,..., <srcM>: The (move to ARISE) instruction is used to support ARISE operations with more input arguments than the available read ports of the processor register file. It moves the contents of registers src1...srcM to the Input Buffer. $M$ is equal to the number of register file read ports. The data are stored in the register of the register bank indicated by the address of the address generation unit. After the store the address is incremented to point to the place where the next data will be stored.

movfa < src1 $>$,..., $<$ srcM $>$ : The (move from ARISE) instruction is used to support ARISE operations with more output arguments than the available write ports of the processor register file. It moves the results of an ARISE operation from the Output Buffer to registers dest1...destN. $\mathrm{N}$ is equal to the number of register file write ports. The data are read from the register of the register bank indicated by the address of the address generation unit. After the read the address is incremented to point to the place from where the next data will be read.

\section{F. Programming an ARISE Machine}

The procedure of programming an ARISE machine starts from the identification of code fragments which can be implemented in a CCU as ARISE operations. What follows is the generation of the configuration bitstreams for these ARISE operations. A configuration bitstream is composed of two parts: the CCU specific and the ARISE specific. The first part is the common bitstream which configures a reconfigurable hardware to implement a certain operation and is generated by the CCU's vendor tools. This bitstream can be generated at any time and ported later to any ARISE machine. Obviously, it is omitted for a hardwired CCU with no need of configuration.

The ARISE specific part holds information which will drive the AIF to successfully execute the operation. For example the number of cycles required for the completion of the operation is part of the ARISE specific configuration bitstream. Only this part must be re-generated each time the operation is implemented in a new ARISE machine. The ARISE specific part of the configuration bitstream is stored in the ACW in a storage structure designed specifically to support the attached CCU. Thus, if a range of addresses has been assigned to a $\mathrm{CCU}$, implying that multiple operations can be configured at the same time in the $\mathrm{CCU}$, the storage structure must support all this different configurations. Furthermore, in the case of a hardwired CCU with standard specifications, the bitstream can be permanently stored in the ACW (firmware can be used instead to insure portability).

The configuration bitstream can be assigned in a high-level data structure which is included in the source code of the application. During linking of the application, the data structure is stored in the main data memory or to a reserved space in a dedicated configuration memory. In the following what remains is the trivial process of constructing the instruction sequences for configuring and executing the ARISE operations. The first is constructed by assigning the opcode of an ARISE operation to a CCU address (opc2addr ARISE instruction) and then - if required - downloading the configuration bitstream (using the confld ARISE instruction). Then, this operation can be called implicitly through its opcode, in any region of the application, until another opcode-to-address assignment invalids it. This call is performed with an instruction sequence of movta-execa-mofa ARISE instructions

\section{AN ARISE EXTENDED MIPS-I EVALUATION MODEL}

To evaluate the proposed scheme a model of an ARISE extended processor has been designed. For this purpose a MIPS with a MIPS-I ISA was used as the core processor under extension. In the following we discuss issues encountered in designing this model. Although a specific processor is used, as it will become clear in the following, the ARISE microarchitecture and the ARISE ISA can be viewed as a general template which can be easily re-targeted to any processor.

\section{A. Encoding the ARISE ISA into the processor instruction word}

The first step in order to create an ARISE extended processor is to encode the ARISE ISA in the instruction word of the processor and design the AID. As already stated the ARISE is tightly-coupled to the processor following the model of a functional unit (just like an ALU is used by a RISC processor). Exploiting this feature the MIPS-I R-Type instruction format is used to encode the ARISE ISA. Figure 3 
shows the R-Type instruction format and the correspondence of the word fields to the ARISE.

\begin{tabular}{|c|c|c|c|c|c|}
\hline 26 & 21 & & & & 5 \\
\hline opcode & rs & rt & rd & shamt & funct \\
\hline ARISE reserved & $\begin{array}{l}\text { src1/opcode/ } \\
\text { start_addr }\end{array}$ & $\begin{array}{l}\text { src2 } \\
\text { ccu_addr/ } \\
\text { end_addr }\end{array}$ & dest & $\begin{array}{c}\text { ARISE } \\
\text { Instruction }\end{array}$ & $\begin{array}{l}\text { ARISE operation } \\
\text { opcode }\end{array}$ \\
\hline
\end{tabular}

Figure 3. Creating ARISE instructions by exploiting the MIPS-I R-Type instruction format.

Only one opcode value must be reserved by the ARISE from the corresponding field of the instruction word. This feature makes possible the extension of architectures with limitation in the number of free opcodes. The ARISE instructions are encoded in the field "shamt" of the MIPS instruction format. This field is five bits long providing a space for $10^{5}=32$ instructions. This number is sufficient for the complete ARISE ISA and possible future extensions. The field "funct" is used to explicitly encode ARISE operations directly in the instruction word of the machine. This field is six bits long giving the possibility to encode $10^{6}=64$ different ARISE operations. If more ARISE operations must be used in a single application space then the dynamic opcode-to-address assignment feature can exploited. Communication of arguments for all ARISE instructions are performed through the processor register file. For this reason the addresses for the two sources (rs, rt) and one destination (rd) registers are used. Since two sources are available, the corresponding instruction variants from the ARISE ISA are used utilizing the complete register file bandwidth.

As already mentioned the AID receives the fetched instruction word and performs an initially decode. If the opcode of the instruction belongs in the processor ISA the instruction word is forwarded as it is to the processor. In the case where the ARISE reserved opcode is identified, the fields "ARISE Instruction" and "ARISE Operation Opcode" are forwarded to the AIF to drive the ARISE operation. In addition the AID modifies the word to form a pseudo-instruction which is part of the processor ISA and will drive the processor operation based on the AIF needs. To achieve this AID replaces the "opcode" and "funct" fields to encode an R-Type instruction which does not raise any exception (i.e. a logical operation). The values of $\mathrm{rs}$, rt, and rd are kept as it is to communicate data with the processor register file. Issuing this pseudo-instruction to the processor will drive the register file to provide the rs and $\mathrm{rt}$ registers and write the result of the operation to the rd register. Thus, the AIF can directly access the rs and rt registers while a multiplex can force the rd value to be provided by the AIF rather than the processor pipeline. Data hazards are resolved by the processor infrastructure.

Table I summarizes the usage of the instruction word fields for each ARISE instruction. When an argument is not required by the ARISE instruction its address is set to the zero register (r0). This register is never written so no data forwarding or stall is performed due to possible data hazard.

\section{B. The ARISE Extended MIPS Compiler and Simulator}

The compiler of the ARISE MIPS-I processor is based on the GNU compiler tool-chain [2]. The tool-chain has been cross-compiled targeting the MIPS processor while the binary utilities have been extended to identify the ARISE instructions. Calling the ARISE is performed directly from the application source code utilizing the inline assembly feature of the GNU compiler. The configuration bitstreams for the reconfigurable CCUs are also encoded directly in the application code using unsigned integer arrays which are declared as global constant variables. Using this tool-chain the targeted application including the ARISE directives are compiled to produce an elf executable.

TABLE I. ENCODING OF THE ARISE ISA IN THE INSTRUCTION WORD FIELDS

\begin{tabular}{|c|c|c|c|c|}
\hline Instruction & Rs & rt & rd & $\begin{array}{c}\text { Operation } \\
\text { Opcode }\end{array}$ \\
\hline confopc2addr & Opcode & $\begin{array}{c}\text { CCU } \\
\text { address }\end{array}$ & r0 & Not Used \\
\hline $\begin{array}{c}\text { confld / } \\
\text { confld_conc }\end{array}$ & $\begin{array}{c}\text { Config. } \\
\text { start } \\
\text { address }\end{array}$ & $\begin{array}{c}\text { Config. } \\
\text { end } \\
\text { address }\end{array}$ & r0 & Not Used \\
\hline execa & src1 & src2 & dest1 & Opcode \\
\hline execa_conc & src1 & src2 & r0 & Opcode \\
\hline movta & src1 & src2 & r0 & Not Used \\
\hline movfa & $\mathrm{r} 0$ & $\mathrm{r} 0$ & dest1 & Not Used \\
\hline
\end{tabular}

The simulator of the ARISE machine is based on the ArchC architecture description language [3]. The MIPS-I ArchC model provided by the ArchC team has been extended with the complete ARISE features and an ARISE MIPS-I simulator was generated. To model the execution of code fragments on CCUs a functional simulation approach was followed. These code fragments were removed from the application code and were placed in a separate source code which was compiled as a dynamic library. This library is imported by the ArchC simulator dynamically to functionally simulate the CCU operation. The simulator has been also extended to provide profiling results regarding the ARISE operation.

\section{EXPERIMENTAL RESULTS}

To demonstrate the effectiveness of our proposal and evaluate its performance we experimented with the ARISE MIPS-I evaluation model and a turbodecoder algorithm as the benchmarking application. The application source code was obtained from the XiRisc benchmarking suite [4]. For the implementation of the application in the ARISE machine we used information derived from [5] where the same application is implemented in the XiRisc [6] reconfigurable processor. Based on this information we assumed that:

- A unit much like the pico Gate Array [5] (p GA) is attach in the ARISE machine as a CCU. This is a reconfigurable LUT-based FPGA-like hardware unit with multiple configuration contexts.

- We identify six different operations in the application code which can be accelerated by implementing them in the pGA CCU as ARISE operations. These operations and the latency of each one in terms of processor cycles are presented in Table II. We further assumed that each operation requires the complete area space to be implemented. 
- The number of cycles required to download the configuration bitstream for a pGA context is 1000 . With 32-bits memory bandwidth for the ARISE machine 32.000 bits are required to configure one pGA contexts.

TABLE II. TURBODECODER OPERATIONS IMPLEMENTED AS ARISE OPERATIONS AND THEIR LATENCY

\begin{tabular}{|c|c|}
\hline ARISE Operation & Latency (Cycles) \\
\hline Butterfly & 7 \\
\hline ComputeGamma & 3 \\
\hline Maxstart & 5 \\
\hline ReorderA & 2 \\
\hline ReorderB & 2 \\
\hline Sum3 & 3 \\
\hline
\end{tabular}

In addition, concurrent operation of the ARISE and the processor was not considered since it requires compiler support which is not yet available. Access of the ARISE to the data memory was also not considered since the implementation of the six operations use only input/output arguments from the processor register file. In both cases with and without ARISE support the source code was compiled with level of optimization set to 2. Finally, when no ARISE is used the six identified operations are called inline and not as functions to produce more fair results.

With this experimental set up a number of different test cases were performed and results are presented in the following.

The first set of our experiments aim to estimate the performance improvements provided by our approach and reveal any possible drawbacks. In this first set we assumed that the CCU supports six configuration contexts. Thus, configuration of operations is performed one time in the start of the application and the operation can then be called without extra overhead. Table III presents experimental results obtained using different combinations of operations. We first simulated the application without using any ARISE operation. Then we performed six times the simulation each time with a different operation used. Finally, we combined the six operations and resimulate the application. The speedup factor shows performance improvement over the case were the ARISE is not exploited. The "Saved Cycles" column gives the benefit from the use of the specific ARISE operation.

Results show that speedup achieved by the use of each operation alone is small. However, when all operations are combined a remarkable $\mathrm{x} 7.52$ speedup is obtained. For this last case Table IV presents the execution cycles spend by each type of ARISE instructions. As it is presented the $57 \%$ of the total execution time is consumed by ARISE instructions. From these cycles execution and configuration ARISE instructions consume most of the execution time. The six ARISE operations have been initially designed to be implemented on the XiRisc processor. However, XiRisc suffers from a 4 inputs 2 outputs arguments limitation. Although, the ARISE machine has a 2 $\mathrm{read} / 1$ write register file exploiting the move instructions it is able to perform these operations. As it is observed only a small
$9 \%$ of the total execution time is consumed in moving data between the ARISE and the processor.

\section{TABLE III. Simulation Performance Results}

\begin{tabular}{|c|c|c|c|}
\hline Operation Used & $\begin{array}{c}\text { SpeedUp } \\
\text { Factor }\end{array}$ & $\begin{array}{c}\text { Execution } \\
\text { Cycles }\end{array}$ & $\begin{array}{c}\text { Saved } \\
\text { Cycles }\end{array}$ \\
\hline Non & 1 & 200168 & NA \\
\hline Butterfly & 1.5 & 132702 & 67466 \\
\hline ComputeGamma & 1.02 & 196353 & 3815 \\
\hline Maxstart & 1.16 & 172836 & 27332 \\
\hline ReorderA & 1.04 & 192835 & 7333 \\
\hline ReorderB & 1.04 & 192428 & 7740 \\
\hline Sum3 & 1.4 & 142051 & 58117 \\
\hline All & 7.5 & 26616 & 173552 \\
\hline
\end{tabular}

In order to experiment with the dynamic reconfiguration capability of the ARISE machine we performed another test case. We assumed that there are only four configuration contexts available in the CCU. This means that not all six operations can be simultaneously configured in the CCU. By careful examination of the application code we conclude that the operations are used exclusively in the functions Branch and $L L R$. In addition, Butterfly operation is used in both functions while ComputeGamma and ReorderB are used only in Branch function and ReorderA, Maxstart, and Sum3 are used only in LLR. So we dedicate one configuration context to the Butterfly operation which was configured at the start of the application. The other three contexts were reconfigured with the appropriate operations before the program flow enters the Branch and LLR respectively.

Table $\mathrm{V}$ presents the execution cycles reported by the simulator for this case. As it was expected the cycles spend for ARISE move and execute instructions remain the same. However, they are now accounting for a smaller portion of the total execution time. This is because execution time consumed in reconfiguring the $\mathrm{CCU}$ has been significantly increased to the $35 \%$ of the total execution time. Increase in the configuration overhead had an impact on the speedup factor which has been reduced from x7.5 to x6.3. However, performance improvements by the exploitation of the ARISE are still significant.

TABLE IV. EXECUTION CYCLES SPENDED For ARISE INSTRUCTIONS

\begin{tabular}{|c|c|c|}
\hline $\begin{array}{c}\text { Instruction } \\
\text { Type }\end{array}$ & $\begin{array}{c}\text { Cycles } \\
\text { Spend }\end{array}$ & $\begin{array}{c}\text { \% of } \\
\text { Total } \\
\text { Cycles }\end{array}$ \\
\hline Move & 2124 & 8 \\
\hline Execution & 6970 & 26 \\
\hline Configuration & 6006 & 23 \\
\hline ARISE Total & 15101 & 57 \\
\hline Program Total & 26616 & 100 \\
\hline
\end{tabular}

\section{RELATED WORK}

Several paradigms of processors augmented by reconfigurable hardware have been presented in the literature. Numerous examples, classification and taxonomy can be found in [1], [13], and [14]. 
TABLE V. EXECUTION CYCLES SPENDED FOR ARISE INSTRUCTIONS ASSUMING LIMITED CONFIGURATION CONTEXTS

\begin{tabular}{|c|c|c|}
\hline $\begin{array}{c}\text { Instruction } \\
\text { Type }\end{array}$ & $\begin{array}{c}\text { Cycles } \\
\text { Spend }\end{array}$ & $\begin{array}{c}\text { \%of } \\
\text { Total } \\
\text { Cycles }\end{array}$ \\
\hline Move & 2124 & 7 \\
\hline Execution & 6970 & 22 \\
\hline Configuration & 11011 & 35 \\
\hline ARISE Total & 20106 & 64 \\
\hline Program Total & 31660 & 100 \\
\hline
\end{tabular}

Prisc [7] uses a RISC processor core augmented with a Programmable Functional Unit (PFU). The unit is tightly coupled to the processor just like a typical ALU. PFU can execute 2 input/1 output operations. Reconfiguration is performed via exceptions. Only one instruction set extension is required to access the PFU. OneChip [8] extends the PRISC allowing the PFU to implement multi-cycle sequential and combinational operations.

Garp [9] is a MIPS processor extended by a custom designed reconfigurable unit. The MIPS instruction set is extended with several instructions in order to control the unit operation following a co-processor-like model. Chimaera [10] like Garp couples a custom designed FPGA-like unit to a MIPS processor. However, the coupling is tighter following the functional unit approach. Communication of data is performed via a shadow register file.

PipeRench [11] augments a core processor with a coarsegrain reconfigurable array of ALUs. PipeRench is focused on implementing linear pipelines of arbitrary length. This machine targets to serve as a co-processor in a general purpose computer and access the same memory space with the host processor.

In Molen [12] instructions are decoded by an arbiter determining which unit is targeted. "Normal" instructions are computed by the hardwired core processor while reconfigurable instructions are computed on the reconfigurable logic. Using this arbiter the processor ISA does not need to be redesigned to support the reconfigurable unit. Tasks to be mapped on the programmable hardware unit are considered microcoded in the processor architecture. The Molen can be considered as a general machine organization allowing a high degree of freedom in the definition of the reconfigurable hardware structure.

XiRisc [6] couples a very-long instruction word (VLIW) processor, featuring a set of digital signal processing (DSP)specific hardwired function units, with a custom designed gate array. The gate array is tightly integrated within the CPU instruction set architecture, behaving as part of both the control unit and the datapath. Reconfigurable instructions implemented in the array can have at most four inputs and two outputs.

In [15] a coarse-grain reconfigurable functional unit is tightly-coupled to a RISC processor. The functional unit consists of a 1-D array of processing elements. The integration of the functional unit to the processor allows the efficient exploitation of the processor pipeline stages. Reconfigurable instructions implemented in the hybrid system combine spatial and temporal computation to speedup execution. The processor register file has been extended to provide four input arguments to the functional unit. In [16] the same architecture has been extended with a "virtual" opcode technique to alleviate the opcode explosion problem and the ability to execute partial predicated operations in the functional unit to improve performance.

As becomes clear by the discussed related work most approaches in the literature present an ad-hoc solution to couple a specific reconfigurable unit with a typical processor. In contrast to that, our approach proposes a general machine organization, a micro-architectural and, an architectural extension which can be systematically performed in any processor. After this extension has been one-time performed, the new ARISE machine can support arbitrary number and any type of reconfigurable units. From this point of view our approach is more related to [12]. In addition, our approach also deals with limitation of previous approaches. Thus, it does not suffer from parameters limitation like PRISC, XiRisc and OneChip, and [15] or opcode space explosion like Chimaera and XiRisc.

\section{CONCLUSIONS}

In this paper, we introduced the ARISE framework for reconfigurable instruction set extensions. The microarchitectural interface of the ARISE allows to a processor to couple arbitrary numbers and types of CCUs. Using the ARISE ISA the programmer/compiler has the full control over the interface and the coupled units. Furthermore, the new instructions can be incorporated without any re-design of the processor ISA. A buffering mechanism is used in order to overcome the parameters limitation problem. Thus, operations with any number of input/output parameters are supported by the ARISE. In addition, using dynamic assignement of opcode to ARISE operations the opcode space explosion problem is also relaxed.

\section{ACKNOWLEDGMENT}

This work was supported by PENED 2003 programme of the General Secretariat for Research and Technology of Greece and the European Union.

\section{REFERENCES}

[1] F. Barat, R. Lauwereins, and G. Deconinck, "Reconfigurable Instruction Set Processors from a Hardware/Software Perspective," in IEEE Trans. Softw. Eng. 28, 9, pp. 847-862, 2002.

[2] http://www.gnu.org

[3] http://www.archc.org

[4] http://www.micro.deis.unibo.it/ campi/XiRisc/Qrisc/software/Programs. $\operatorname{tg} z$

[5] A. La Rosa, L. Lavagno, and C. Passerone, "Software Development for High-Performance, Reconfigurable, Embedded Multimedia Systems," in IEEE Design and Test of Computers, vol. 22, no. 1, pp. 28-38, 2005.

[6] A. Lodi, M. Toma, F. Campi, A. Cappelli, R. Canegallo, and R. Guerrieri, "A VLIW Processor With Reconfigurable Instruction Set for Embedded Applications," IEEE Journal Of Solid-State Circuits, Vol. 38, No. 11, pp. 1876-1886, November 2003

[7] R. Razdan and M.D. Smith, "A High-Performance Microarchitecture with Hardware-Programmable Functional Units," Proc. 27th Int'l Symp. Microarchitecture (MICRO 27), pp. 172-180, Nov. 1994. 
[8] R.D. Wittig and P. Chow, "OneChip: An FPGA Processor with Reconfigurable Logic," Proc. Workshop FPGAs and Custom Computing Machines (FCCM '96), pp. 126-135, 1996.

[9] J.R. Hauser and J. Wawrzynek, "Garp: A MIPS Processor with a Reconfigurable Coprocessor," Proc. Workshop FPGAs and Custom Computing Machines (FCCM '97), pp. 12-21, 1997.

[10] S. Hauck, T. Fry, M. Hosler, and J. Kao, "The Chimaera Reconfigurable Functional Unit," Proc. Workshop FPGAs and Custom Computing Machines (FCCM '97), pp. 87-96, 1997.

[11] S.C. Goldstein, H. Schmit, M. Moe, M. Budiu, S. Cadambi, R.R. Taylor, and R. Laufer, "PipeRench: A Coprocessor for Streaming Multimedia Acceleration," Proc. 26th Int'l Symp. Computer Architecture (ISCA '99), pp. 28-39, May 1999.

[12] S. Vassiliadis, S. Wong, G. N. Gaydadjiev, K. Bertels, G.K. Kuzmanov, E. Moscu Panainte, The Molen Polymorphic Processor, IEEE Transactions on Computers, pp. 1363- 1375, November 2004, Volume 53 , Issue 11 .
[13] K. Compton and S. Hauck, "Reconfigurable Computing: A Survey of Systems and Software," ACM Computing Surveys, vol. 34, no. 2, pp. 171-210, 2002

[14] M. Sima, S. Vassiliadis, S Cotofona, J. van Eijndhoven, and K.Vissers, "Field-Programmable Custom Computing Machines - A taxonomy," Proc. 12th Int'l Conf. Field Programmable Logic and Applications (FPL), pp. 79-88, Sept. 2002.

[15] N. Vassiliadis, N. Kavvadias, G. Theodoridis, S. Nikolaidis, "A RISC architecture extended by an efficient tightly coupled reconfigurable unit," International Journal of Electronics, Volume 93, Number 6 / June 2006, p. 421 - 438.

[16] N. Vassiliadis, G. Theodoridis, S. Nikolaidis "Enhancing a Reconfigurable Instruction Set Processor with Partial Predication and Virtual Opcode Support", in Proceedings of the International Workshop on Applied Reconfigurable Computing (ARC2006), DELFT, The Netherlands, March 1-3, 2006. 